\title{
Liquid-phase imaging of bone development and calcification by atmospheric scanning electron microscopy (ASEM): Application to immuno-labeling and rapid tissue observation of genetically modified mouse
}

\author{
Eiko Sakai ${ }^{1}$, Mari Sato ${ }^{2}$, Nassirhadjy Memtily ${ }^{3}$, Takayuki Tsukuba ${ }^{1}$ and Chikara Sato ${ }^{4}$
}

${ }^{1}$ Division of Dental Pharmacology, Department of Developmental and Reconstructive Medicine, Nagasaki University Graduate School of Biomedical Sciences, Nagasaki 852-8588, Japan, United States, ${ }^{2}$ Health and Medical Research Institute, National Institute of Advanced Industrial Science and Technology (AIST), Tsukuba, Ibaraki 305-8566, Japan, United States, ${ }^{3}$ Traditional Uyghur Medicine Institute of Xinjiang Medical University, Xinjiang Uyghur Autonomous Region, Urumqi 830011, China, Urumqi, Xinjiang, China (People's Republic), ${ }^{4}$ Health and Medical Research Institute, National Institute of Advanced Industrial Science and Technology (AIST), Tsukuba, Ibaraki 305-8566, Japan[, United States

Bone seems like a solid mass of calcium phosphate, but inside it, unique cells such as chondrocytes, osteoblasts, osteocytes, and osteoclasts are densely present and cooperate with each other to grow and repair bone [1]. The development of long bones such as the femur and tibia is controlled through a finely regulated mechanism of chondrogenesis and angiogenesis called endochondral ossification. In the cartilage tissue at both ends of long bone, depending on the differentiation stage, round-shaped restingchondrocytes become proliferating-chondrocytes, and finally differentiate into hypertrophicchondrocytes. They secrete cartilage matrix around them. After deposition of calcium phosphate, terminal hypertrophic-chondrocyte die and blood vessels are penetrated to the emptied hypertrophic-chondrocyte zone. Progenitors of osteoblasts and osteoclasts invade this area by vascular invasion. [2].

Using atmospheric scanning electron microscopy (ASEM) (Fig. 1A), we succeeded in high-resolution observation of mineral-containing bone and surrounding cells without decalcification, dehydration, and thin-sectioning. Since the specimens were mineralized tissues, the calcified parts showed bright images when we directly observed the aldehyde-fixed tissues without phosphotungstic acid (PTA) staining [3]. By this method, we were able to easily clarify the impaired calcification of gene-knockout mouse femurs of Keap1, an oxidative-stress sensor and negative regulator of Nrf2 [4].

Consistent with the previous studies that the trabecular bone area is not yet calcified at embryonic 15.5 days, no mineralization was detected by ASEM without PTA staining. However, chondrocytes and surrounding cartilage matrix were clearly observed after PTA staining. At postnatal day 1, bright images were observed even without PTA staining, suggesting the initiation of calcification. After PTA staining, the filamentous structure between osteoblast-like cells and osteocytes surrounded by cortical bone were clearly imaged. In addition, by immunolabeling using a specific antibody against cathepsin $\mathrm{K}$, which is a marker enzyme of osteoclasts, and osteoclast-like cells with ruffled border extended to the bone were successfully identified (Fig. 1B). Numerous different shaped cells and resorption pits were imaged in hypertrophic-chondrocyte and trabecular zone at postnatal day 1 and day 6 (Fig. 1C, 1D).

Keap1 gene-deficient mice exhibit growth retardation and juvenile lethality [4]. In Keap1-deficient mice femur at postnatal day 6, we revealed delayed hypertrophy and vascular invasion of resting-chondrocyte zone by ASEM, suggesting impaired secondary ossification center formation (Fig. 2A-C) [5]. Our results indicate that this high-throughput technology is extremely effective for observing bone metabolism during 
the development of hard tissues. Particularly, it can be expected to be widely applied to the observation of hard tissues of various gene-deficient mice exhibiting a juvenile lethal phenotype.

Fig. 1A Atmospheric SEM (ASEM)
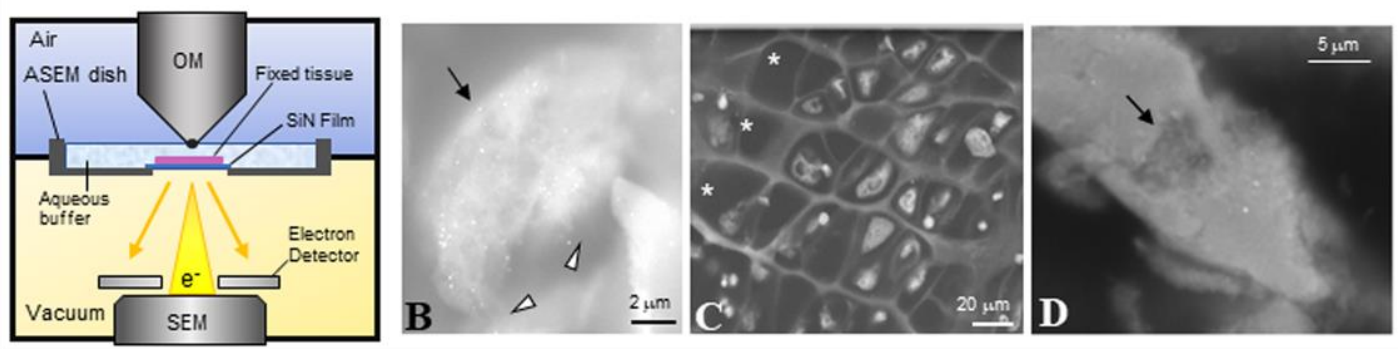

Figure 1. ASEM enables observation of hard tissue in liquid and identification of osteoclasts by immunogold labeling. (A) ASEM system used in this study. (B) At postnatal day 1, wild-type mouse femur was aldehyde-fixed, labeled with anti-cathepsin K antibody and further with FluoroNanogold conjugatedsecondary antibody, gold-enhanced, and then counter-stained with PTA. A cathepsin K positive osteoclast-like cell (arrow) with ruffled border (arrowheads) was identified. (C) In hypertrophicchondrocyte zone, hypertrophied chondrocytes, round-shaped blood cells, and empty lacunae $(*)$ were imaged. (D) Arrow indicates the resorption pit on the mineralized trabecular bone.
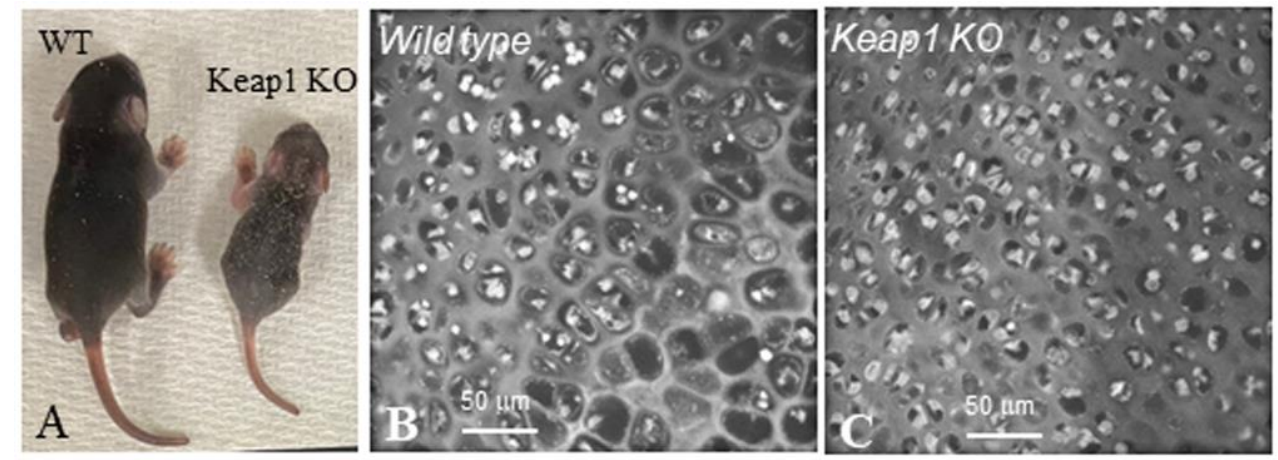

Figure 2. The quick ASEM method revealed impaired bone development in Keapl knockout mouse. (A) At postnatal day 6, Keapl KO mouse exhibits growth retardation. (B) In the epiphyseal chondrocyte zone, cells were hypertrophied in wild-type femur, but not in Keapl KO mouse (C).

References

[1] G. Karsenty, Nature 423, 316-318 (2003)

[2] H.M. Kronenberg, Nature 423, 332-336 (2003)

[3] C. Sato, et al, Sci Rep 9, 7352 (2019)

[4] N. Wakabayashi, et al, Nat. Genet. 35, 238-245 (2003)

[5] E. Sakai, et al, Sci Rep (2021) doi: 10.1038/s41598-021-84202-z 